\title{
Contexto Institucional para la Introducción de la Evaluación Ambiental Estratégica (EAE) en Colombia
}

Jorge Gómez Duque, Doktor-Ingenieur*

nestropico@hotmail.com

\section{RESUMEN}

Las posibilidades de introducir la Evaluación Ambiental Estratégica (EAE) en Colombia (la cual se aplica a nivel de políticas, planes y programas-PPP), ${ }^{2}$ implica analizar el contexto sociopolítico, los procesos de gestión y en general el entorno en que se desarrollan las políticas públicas, aún más considerando la variedad, entornos específicos, formas particulares de estructuración formal, diferentes modalidades de interdependencias, etc. de las políticas, planes y programas existentes en diferentes ámbitos.

\section{Palabras Claves:}

Evaluación Ambiental Estratégica, Políticas Públicas, Proceso de Toma de Decisiones, Ciclo Político, Proceso de Planificación.

* Profesor Especialización en Educación y Gestión Ambiental, Facultad de Ciencias y Educación. neotropico@hotmail.com,jegomezd@udistrital.edu.co

La Evaluación Ambiental Estratégica (EAE) es entendida como la evaluación ambiental sistemática, aplicada en las etapas tempranas del proceso de planificación anteriores a la de Proyecto, es decir, a nivel de Políticas, Planes y Programas (PPP). A diferencia de la EAE, la Evaluación de Impacto Ambiental, se aplica: en el proceso final de planificación, o sea, en el de Proyectos. 


\section{ABSTRACT}

The possibilities of introducing Strategic Environmental Assessment (SEA) in Colombia (which is applied to level of policies, plans and programs-PPP), it implies to analyze the social-politic context, the processes of management and in general the background in which the public policies are developed, still more considering the variety, specific circumstances, particular forms of formal structuring, different modalities from interdependences, etc. of the existing policies, plans and programs in different scopes.

\section{INTRODUCCIÓN}

En general el diseño de la EAE apropiada para un país, debe considerar diferentes determinantes de la sociedad en que se va a aplicar, para que tenga viabilidad su adopción. Cupei (1994)², advierte sobre el peligro de hacer.refinámientos y optimizaciones de modelos de Evaluación Ambiental de acuerdo a conceptos ideales. Entre los factores condicionantes que se deben considerar para este diseño están las características del sistema político administrativo como la tradición y cultura jurídica, la estructura y formas de relación político-administrativas, la cultura política (relaciones ciudadanoEstado, relaciones ciudadano-economía, relaciones entre el Estado y la investigación, confianza en el Estado, sistema de valores en generall), sin omitir las condiciones geográfico-naturales del país. Partiendo de estas condiciones y marco generales se deben concebir los modelos concretos de EAE y su optimización.

\section{CONDICIONES MARCO EN EL PAÍS OUE CONDICIONAN LA ESTRUCTURACIÓN DE LA EVALUACIÓN AMBIENTAL ESTRATÉGICA (EAE)}

Con relación a un país como Colombia, podrían considerarse aspectos relevantes sobre . esas condiciones marco, los siguientes ${ }^{3}$ :

\subsection{Condiciones Geográfico-Naturales}

Diversidad y heterogeneidad de los ecosistemas, Capacidad de resiliencia ecológica, Área en ecosistemas naturales conservados y área en ecosistemas transformados, Topografía y facilidad de acceso de los diferentes sitios y regiones, etc.

\subsection{Tradición y Cultura Jurídica}

Desarrollo específico de la normatividad ambiental -más bien de acuerdo a normas generales o de acuerdo a normas específicas; Coordinación y repartición de compe-

CUPEI, Jürgen "Umweltverträglichkeitsprüfung und Entscheidung" en Vorsogen statt nur Schaden Beseitigen. Dortmund: Verein zur Förderung der UVP, 1994

3 Apoyándose en marcos teóricos sobre la Evaluación de Impacto Ambiental de CUPEI (Op. Cit.) y de GÓMEZ, Jorge "Entwicklung der Umweltpolitik in Kolumbien dargestellt am Beispiel der Umweltverträglichkeitsprüfung" Berlín: Universidad Técnica de Berlín, 1997 
tencias para el desarrollo y aplicación de la normatividad entre las diferentes instituciones y niveles territoriales; Déficit en la aplicación de la reglamentación ambiental o bien, cumplimiento de la misma, etc.

\subsection{Estructura y Cultura Administrativa-Institucional}

Evolución de la estructura administrativo institucional; Posición y jerarquía de la instituciones responsables de la gestión ambiental en el aparato institucional, Repartición de funciones entre las diferentes instituciones responsables de la gestión ambiental, Dotación institucional, Comportamiento habitual de las instituciones con relación a otras instituciones del aparato político-administrativo, etc.

\subsection{Cultura Política}

- Relación Estado-Economía: Determinantes principales de la acción estatal con respecto a la política económica, social y ambiental; Participación del Estado en las actividades económicas, Tipo de relaciones entre el Estado y los grupos económicos, y por tanto las relaciones entre Estado-Economía-Sociedad civil. De acuerdo a esta situación se caracteriza la cercanía del Estado a la iniciativa de los sectores económicos, de los ciudadanos, de las organizaciones en el área ambiental, así como se estructuran las exigencias ambientales y las respuestas del Estado a estas, etc.

- Relación Estado-Sociedad Civil: Formas de interacción entre Estado y la Sociedad Civil; Cultura de participación; Contexto social e integración y comportamiento para hacer rendición de cuentas del Estado en las diferentes áreas y en la ambiental específicamente, etc.

- Relación Estado - Investigación: Apoyo a la investigación para la gestión ambiental en general y para la aplicación de instrumentos de gestión ambiental; Papel de la ciencia en el desarrollo de la gestión ambiental.

- Cultura del Riesgo y Sistema de Valores: Cultura en la sociedad para tratar los riesgos, incertidumbres y vacíos de información en el área ambiental; Cultura de transparencia con relación a las actuaciones del Estado; Forma de comportarse del Estado frente a conflictos entre la economía y lo ambiental, etc.

Los factores anteriores se deben tener presente como transfondo para el análisis del modelo de evaluación ambiental estratégica que se puede implementar en Colombia. Sin embargo los más determinantes en la estructuración de la EAE son los de tipo político administrativo. Ya que la EAE se aplica a Políticas, Planes y Programas, el análisis de este ámbito estratégico del proceso de decisiones es muy relevante, es decir, se requiere profundizar sobre aspectos específicos de la estructura y el proceso político administrativo como el ciclo político y el contexto sociopolítico de los PpP; la naturaleza de las decisiones estratégicas; características y organización de los instrumentos de planificación; tipos, contenidos, gestión de PPP; etc. Estos se pueden considerar como determinantes para definir el modelo de EAE que se puede introducir en el país, teniéndose también como referencia el marco para la gestión ambiental existente. 


\section{CARACTERIZACIÓN GENERAL DEL PROCESO DE TOMA DE DECISIÓN A NIVEL DE LAS POLÍTICAS, PLANES Y PROGRAMAS (PPP| ${ }^{4}$}

Para Nilsson y Jiliberto (2004), la mayoría de los procesos de decisiones públicas no se pueden explicar de acuerdo a un modelo racionalista. En general el nivel de racionalidad involucrado en la toma de decisiones podría estar correlacionado con el nivel de abstracción de la decisión, es decir, con su nivel de jerarquía, en el proceso de PPP, que termina con el nivel de proyectos. Los niveles de decisión más elevados parecieran ser más difíciles de caracterizar con el modelo racional de toma de decisiones que los niveles más concretos, por ej. el de proyectos de inversión a nivel local. Por eso el modelo racional es una pobre representación de la toma de decisiones en el nivel estratégico. En lugar del enfoque anterior, se deberían de considerar otros factores en el centro de este proceso como los intereses de los diferentes actores, el poder y los valóres involucrados en la decisión. Max Weber decía que cada acción (y en cada acción) se podía reconocer la aceptación de ciertos valores y el rechazo de otros (Duna, 1994). En estos procesos se trata con sistemas que se caracterizan por su complejidad debido al múltiple involucramiento de actores y también por sus complicaciones técnicas.

No obstante lo anterior (siguiendo a Nilsson y Jiliberto, Op. Cit.), el proceso de toma de decisiones en todos los niveles comparte rasgos de las diferentes teorías sobre la toma de decisiones. Por ejemplo las decisiones públicas se necesitan basar en evaluaciones y medidas racionales y objetivas, pero en últimas se toman de acuerdo al valor que le da la sociedad a los asuntos que son discutidos en estas decișiones. Cuando los criterios y valores son hechos explícitos, el proceso de decisión llega a ser transparente y controlable. Para esto se requiere que quien toma las decisiones haga explícita sus escogencias entre las diferentes opciones. No se trata de un asunto sobre si los elementos subjetivos están involucrados o no, sino si ellos están articulados y si se incorporan de una forma sistemática en el proceso. de toma de decisiones.

Los diferentes desarrollos teóricos sobre la toma de decisiones y el análisis de políticas han contribuido a construir un completo cuadro de explicación y descripción del proceso de toma de decisiones que debería servir para el desarrollo de la evaluación ambiental estratégica. Es imposible plantear que el proceso de decisión en un nivel estratégico toma lugar en toda su extensión bajo una única racionalidad. Algunos procesos serán más racionales, otros menos objetivos y cargados de mayor subjetividad. La evaluación ambiental debería de tener en cuenta esto y responder a estas variaciones.

Se podría argüir que si el proceso de toma de decisiones (privado o público), fuera completamente racional, todos los valores y criterios deberían ser considerados, incluyendo los costos y beneficios de cada alternativa. En este caso no debería haber alternativa a la evaluación ambiental. El desarrollo del proceso de esta manera significaría que sería superfluo cualquier intento de hacerlo más eficiente lya que incluye todos los criterios económicos, sociales y ambientales relevantes). Sin embargo la evaluación ambiental se requiere ya que la aplicación de criterios ambientales no sucede en la práctica automáticamente.

4 Basado en NILSSON. Mäns; JILIBERTO, Rodrigo "SEA and Decision-Making Sciencies" en Analysing Strategic Environmental Assessment - Towards Better Decisión-Making. Glos (Gran Bretaña): Edgar Publishing Limited, 2004 
Los procesos de decisión completamente racionales son una construcción básicamente teórica. La evaluación ambiental para que sea efectiva deber ir más allá de la predicción de las consecuencias ambientales de la decisión y asegurar que un conjunto de procedimientos sea aplicado en el proceso de toma de decisiones que garantice que las consideraciones ambientales sean tenidas en cuenta. En ese sentido comparando la EAE (que se aplica a nivel de PPP) con la Evaluación de Impacto Ambiental-EIA (que se adopta en los proyectos, dònde el nivel de concreción teóricamente posibilita una toma de decisiones más racionall, se puede decir que su estructuración (de la EAE) no debe seguir los mismos pasos habituales considerados en la EIA. El contexto en el cual opera es diferente al de la EIA.

\section{CONTEXTO DE POLÍTICAS, PLANES, PROGRAMAS, CARACTERIZACIÓN DEL CICLO POLÍTICO Y DE LOS INSTRUMENTOS DE PLANIFICACIÓN EN COLOMBIA ${ }^{5}$}

En el país es habitual que no haya una diferenciación muy formal entre planes, politicas y programas. Si bien teóricamente se plantea que a nivel de las decisiones estratégicas o de PPP, el nivel de políticas ${ }^{6}$ es el más general, esta organización jerárquica se desdibuja frecuentemente en la práctica. Esta situación puede obedecer a diferentes factores, los cuales siguiendo categorías de análisis generales para el estudio de PPP serían: Ciclo Político y los Procesos de Decisión; Relaciones entre PPP y Diversidad de Contextos Institucionales y de Niveles Territoriales en que se desarrollan los PPP'.

\subsection{Ciclo Político y Procesos de Decisión}

Es importante anotar que el nivel de políticas (comparándolo con el de planes y programas), cumple un papel más ideológico. En este se plantean las directrices generales en un ámbito determinado del desarrollo, de acuerdo a una visión de sociedad específica. Estos lineamientos deberían ser recogidos posteriormente para su operacionalización a nivel de los planes y programas. Muchos de los asuntos que terminan siendo recogidos en los planteamientos de la respectiva política no se da siempre de acuerdo a una racionalidad técnica, sino que pueden depender mucho de los puntos de vista del mandatario de turno, de las presiones e intereses de los partidos y de las clientelas que los apoyan, del momento electoral (los intereses de acuerdo a este momento tienden a ser de tipo cortoplacistas). de las presiones de los gremios y grupos organizados con capacidad de hacer cabildeo, de la urgencias y presiones sociales del momento, de las iniciativas de la burocracia y asesores con peso en la toma de decisiones, etc. En estas circunstancias la formulación y negociación de políticas están menos dominadas por procesos de toma de decisión racionales.

5 Reflexiones del investigador complementadas por su experiencia laboral anterior como funcionario de la administración pública del nivel nacional y distrital, apoyándose en el marco de referencia para este tipo de análisis propuesto por ONATTE, Juan y Otros "La Evaluación Ambiental Estratégica" Madrid: Ediciones Mundiprensa, 2002

- Los lineamientos de una política teóricamente se deben concretar a nivel de un plan, el cual a su vez debería contener diferentes programas que se operacionalizan por medio de diferentes proyectos

7. Marco de referencia propuesto por OÑATE, Juan y Otros (Op. Cit.) 
Por otra parte, en las condiciones de Colombia donde se han abierto canales formales de participación a diferentes actores para la elaboración de estas políticas (planes y programas), pero a su vez, considerando que estos mecanismos muchas veces no han sido ápropiados por estos actores o solo se permite por parte de los tomadores de decisiones una participación formal sin incidencia real en los derroteros del diseño de los PPP, hay mucha más opción para que de una forma poco participativa y no tan transparente, los PPP se diseñen y desarrollen. La existencia de esta gran discrecionalidad por parte de los "detentores" del poder también influye en sesgos para darle la denominación respectiva a los PPP en discusión. Muchas veces a una política se le denomina plan o programa, e incluso hasta se le ha dado el nombre de proyecto ${ }^{8}$, lo cual dificulta la distinción entre ellos.

Las características del entorno en que se gestan las políticas, hace más difícil la aplicación de una evaluación ambiental, instrumento que generalmente se ha diseñado para procesos de toma de decisiones en que predomina una racionalidad más bien de tipo técnico. Por eso su aplicación por lo regular es más favorable realizarla a nivel de los planes y programas, ya que por ser niveles más instrumentales, permiten en principio que prime mucho más que en el nivel de las políticas, esta racionalidad técnica arriba mencionada.

\subsection{Relaciones entre PPP}

En el país no existe una reglamentación y/o acuerdos generales a nivel de la administración pública que permita hacer una clasificación y separación clara entre PPP. Así como existen políticas que por su nivel de concreción parecieran programas, también se dan programas que por su generalidad realmente son políticas. Estos vacíos normativos y falta de acuerdos ha impedido lograr una homogenización relativa sobre terminología, alcances, contenidos, etc. de las políticas, planes y programas, lo cual ha conducido (apoyándose en Oñate y otros, 2002) ${ }^{9}$, a que se encuentre una diversidad de iniciativas con un alta variabilidad de concreción espacial, instrumental, temporal y presupuestal. Con frecuencia no hay una secuencia temporal entre los PPP y su jerarquía entre sí tampoco está siempre clarificada. Solo algunos PPP en áreas específicas pueden tener disposiciones que reglamentan sus contenidos mínimos y el procedimiento administrativo para elaborarlos. Sin embargo esta reglamentación no siempre se hace con cierto detalle, dejando amplias opciones de interpretación. Esa diversidad y confusión, condiciona de una manera muy importante las metodologías que se pueden desarroliar, especialmente en la identificación de los efectos de PPP, en el marco de una evaluación ambiental estratégica.

\subsection{Diversidad de Contextos Institucionales y de Niveles Territoriales en que se desarrollan los PPP}

En el país existen básicamente tres niveles político-administrativos: Nacional, Regional (Departamental) y Local, cada uno con su propia normatividad y cultura institucional.

8 Un ejemplo de esto fue el nombre recibido por la Política Ambiental durante el Gobierno de Andrés Pastrana (1998-2002). Esta se denominó "Proyecto Colectivo Ambiental"

9 OÑATE, Juan y Otros (Op. Cit.) 
La coordinación e interacción entre estos niveles aunque puede estar regulada, no siempre se distingue por la cooperación y armonización en el desarrollo de los PPP según las competencias y responsabilidades fijadas para cada nivel. A esto contribuye también la gestión de las diferentes áreas del desarrollo de forma compartimentalizada, tanto a nivel nacional, regional y local. Varias de ellas como la temática ambiental que se caracteriza por su transversalidad e interacción con las otras áreas, encuentra a menudo limitaciones para ser considerada transversalmente por la forma departamentalizada (es decir, por áreas), como frecuentemente está estructurado el aparato institucional. La fragmentación institucional que comúnmente se presenta en el aparato políticoadministrativo a todos los niveles, tiene su reflejo a su vez en las iniciativas e instrumentos que se manejan en cada uno de ellos, como por ej. las PPP.

Así mismo la capacidad política, técnica, presupuestal entre los diferentes niveles de la escala territorial es diferente. Incluso al interior de cada uno de estos niveles hay diferencias marcadas de capacidades, por ej. entre las mismas instituciones del nivel nacional, entre departamentos y municipios.

La anterior heterogeneidad se constituye en un reto cuando se quiere introducir la EAE. Su institucionalización debe ajustarse a esta realidad institucional desigual y compleja $y$, debe superar las dificultades que representa la fragmentación institucional.

\section{BIBLIOGRAFÍA}

CUPEI, Jurgën “Umweltverträglichkeitsprüfung und Entscheidung” Dortmund: Verein zur Förderung der Umweltverträglichkeitsprüfung, 1994.

GOMEZ, Jorge "Entwicklung der Umweltpolitik in Kolumbien dargestellt am Beispiel der Umweltverträglichkeitsprüfung" Berlín: Universidad Técnica de Berlín, 1997.

NILSSON, Mäns; JILIBERTO, Rodrigo "SEA and Decision-Making Sciencies" en "Analysing Strategic Environmental Assessment - Towards Better Decision-Making" Glos /Gran Bretaña): Edgard Edgar Publishing Limited, 2004.

OÑATE, Juan y Otros "La Evaluación Ambiental Estratégica" Madrid: Ediciones Mundiprensa, 2002. 